\title{
Dynamic Evolution of the Operating Efficiency of Development Zones in China
}

\author{
Shuang Gao ${ }^{1}$, Jingwen Zhang ${ }^{2}$, Xiaoqing $\mathrm{Mo}^{2}$ and Rong $\mathrm{Wu}^{2, *}$ \\ 1 Guangdong Provincial Key Laboratory of Urbanization and Geo-Simulation, School of Geography and \\ Planning, Sun Yat-sen University, Guangzhou 510275, China; gaosh6@mail2.sysu.edu.cn \\ 2 School of Architecture and Urban Planning, Guangdong University of Technology, Guangzhou 510090, China; \\ 3217008157@mail2.gdut.edu.cn (J.Z.); 3217008148@mail2.gdut.edu.cn (X.M.) \\ * Correspondence: wurong@gdut.edu.cn; Tel.: +86-181-2226-6326
}

Citation: Gao, S.; Zhang, J.; Mo, X.; $\mathrm{Wu}, \mathrm{R}$. Dynamic Evolution of the Operating Efficiency of Development Zones in China. Sustainability 2021, 13, 10395. https://doi.org/10.3390/ su131810395

Academic Editor: Fernando Almeida

Received: 2 August 2021

Accepted: 10 September 2021

Published: 17 September 2021

Publisher's Note: MDPI stays neutral with regard to jurisdictional claims in published maps and institutional affiliations.

Copyright: (c) 2021 by the authors. Licensee MDPI, Basel, Switzerland. This article is an open access article distributed under the terms and conditions of the Creative Commons Attribution (CC BY) license (https:/ / creativecommons.org/licenses/by/ $4.0 /)$.
Abstract: The efficient development of Economic and Technological Development Zones (ETDZs) across China has been key to the country's economic transformation. Despite this, few studies have addressed the dynamic characteristics of the efficiency of ETDZs. Taking China's 115 national ETDZs established before 2010 as the research sample, this study measures the comprehensive operating efficiency of ETDZs and identifies dynamic characteristics of that efficiency throughout the period 2011-2017, using a three-stage data envelopment analysis (DEA) model and the Markov method. This research also explores the relationship between the operation efficiency of ETDZs and urban economic development. The results indicate that the operating efficiency of national ETDZs was generally low, and subject to a downward trend over the study period; the efficiency of ETDZs in the western region was found to be higher than that in the central and eastern areas. It was found that operating efficiency was stable in terms of the types of transfer witnessed, and the results emphasize the difficulty of achieving leapfrog development in a relatively short time. In addition, medium-high coupling coordination was detected in the relationship between urban social and economic development and the operating efficiency of ETDZs. When the operating efficiency of the ETDZ was high, this coupling coordination improved significantly. Finally, most of China's ETDZs remain in a factor-driven stage in their development, with their expansion occurring at the expense of efficiency. Based on these results, this paper suggests that the government should pay greater attention to the overall efficiency and growth quality in the sustainable development of the country's ETDZs.

Keywords: economic and technological development zone; operating efficiency; dynamic characteristic; spatiotemporal differentiation; coupling coordination relationship

\section{Introduction}

As a spatial carrier of China's innovation system and a location open to the outside world, the Economic and Technological Development Zone (ETDZ) has played a critical role in developing international trade and attracting foreign investment by means of preferential policies [1-3]. Since the construction of the earliest batch of China's ETDZs in 1984, and following more than 30 years of development, remarkable achievements in industrial cultivation, urban construction, land development, and export promotion have been achieved through implementation of these zones [4,5]. By 2018, China had a total of 219 national ETDZs with an annual GDP of 10.2 trillion CNY, or $11.3 \%$ of the national GDP, and an actual utilization of foreign capital of 51.3 billion USD, accounting for $20 \%$ of the national figure [6]. National ETDZs have become the growth pole of China's economy.

In view of the important role that ETDZs play in Chinese economic development, the effects of this policy have attracted widespread attention from scholars. Early research mainly focused on the positive impact of ETDZ policies on regional development from multiple perspectives, including the promotion of technological progress [7], acceleration 
of capital accumulation [8] and improvement of development efficiency [9]. For example, Wang [10] used an urban-scale municipal dataset to measure the impact of ETDZs on regional development. The empirical results suggested that by attracting foreign capital investment, ETDZs could help cities to realize capital accumulation and promote the regional development economic growth. Moreover, when a city had more than one ETDZ, the process of capital accumulation would be more rapid. Herlevi [11] discussed the role of ETDZs in regional political economy from the perspective of land use. The study found that ETDZs significantly changed regional land use patterns and urbanization levels, thereby promoting regional economic development. In addition to urban-scale research, scholars have also explored the impact of ETDZs on regions from the perspective of enterprises. A study by Huang et al. [12] measured the land utility efficiency of enterprises within and outside ETDZs and carried out a comparative analysis. It found that under the influence of both the agglomeration effect and the factor accumulation effect, the land-use efficiency and land output of enterprises located in an ETDZ were significantly higher than those of enterprises located outside such zones. Sun et al. [13] found that well-managed ETDZs could significantly promote the scale growth and output efficiency of their internal enterprises. In addition, recent studies have shown that not only is there a spatial spillover effect between enterprises within the ETDZ [8], but that this spillover effect spreads to the periphery of the ETDZ and affects urban construction around the ETDZ [14,15].

The various advantages of ETDZs have prompted governments across China to strive for preferential policies to compete for foreign investment, which has led to an explosive growth in the number of ETDZs [16]. This process has been referred to as "zone fever" or "project fever" in academic circles [17]. This rapid expansion of the number and scale of ETDZs has not been without serious problems. ETDZs established by many local jurisdictions never received any investment and sat idle for years as undeveloped sites [18]. "Zone fever" or "project fever", along with lagging administrative reforms, led to corruption and wasteful development in China [19]. Additionally, due to chaotic management, industry convergence, and unclear positioning, most of China's ETDZs have not harnessed potential scale and agglomeration effects, and the phenomena of land waste and inefficient use of funds are both evident $[20,21]$. China is currently at a pivotal juncture of economic transformation, and the focus of the government has changed from the speed of economic development to the quality of economic development [22]; and as the growth pole of China's economy, the effective development of ETDZs is recognized as critical to successful economic transformation. In this context, increased attention must be directed to the task of evaluating the efficiency of ETDZs and determining whether resources are being effectively allocated.

Efficiency in economic activities mainly reflects the effects of the allocation of various resource elements in a specific environment [23]. The measure of "operating efficiency" can be understood as the output effect of the input of resource elements under certain technical conditions-as such, this measure reflects the level of utilization of various resource elements. If an ETDZ has high operating efficiency, this indicates that the allocation of each input factor is reasonable. At present, relatively little research has been conducted on the efficiency of ETDZs or changes in their efficiency, and studies that have addressed this issue have mainly analyzed land-use efficiency and production efficiency of ETDZs [24-28]. The existing research has a number of shortcomings. Firstly, in analyzing the efficiency of ETDZs, the majority of studies have used the stochastic frontier analysis (SFA) model or the data envelopment analysis (DEA) model, methods which are easily affected by the external environment and thus cannot reflect the true level of efficiency in the case of a spatial entity such as an ETDZ [29]. Second, the existing research has mainly consisted of static analyses of the efficiency of ETDZs; to date a dynamic description remains lacking. Third, existing studies have not considered spatial differences in the efficiency of ETDZs.

Attending to the importance of and existing gaps in efficiency research, this paper puts forward the following research framework. Firstly, this study adopted a three-stage model that combined DEA and SFA models to estimate the true level of efficiency of 
ETDZs. Secondly, a Markov chain transfer matrix was constructed to identify the evolution trend and spatial differentiation characteristics of the efficiency of ETDZs in the three major regions of eastern, central, and western China. Finally, a coupling coordination degree model was also employed to explore in detail the dynamic coupling relationship that exists between the operating efficiency of ETDZs and urban economic growth. This paper then used the exploratory analysis described above to propose a reference for policymakers in confronting the task of further optimizing resource allocation and promoting the high-quality development of ETDZs.

\section{Data and Methods}

\subsection{Index System Construction and Data Acquisition}

This study adopted an input-output perspective in order to estimate the operating efficiency of China's national ETDZs from 2011 to 2017 and to identify the coupling and coordination relationship between operating efficiency and urban economic growth. The research sample addressed by the study comprised 115 ETDZs established in or before 2010 . China's ETDZ strategy focuses on industrial projects, attracting investment, and promoting exports. With this in mind and seeking to accurately reflect the operational characteristics of ETDZs, this paper selected the actual utilization of foreign capital, total import volume, and approved area of the ETDZ to constitute the input indicators addressed in this study, and GDP and total export volume as the output indicators. Together, this resulted in a set of input-output indicator systems to estimate the operating efficiency of national ETDZs (Table 1).

Table 1. Input-output indicator systems for China's ETDZs' operating efficiency.

\begin{tabular}{ccccccc}
\hline Indicator & Variable & Unit & Mean & Max & Min & Std. Dev. \\
\hline \multirow{2}{*}{ Input } & actual utilization of foreign capital & $10^{8} \mathrm{CNY}$ & 24.576 & 432.9 & 0.001 & 40.589 \\
& total import & $10^{8} \mathrm{CNY}$ & 171.569 & 2982.84 & 0.001 & 353.157 \\
& volume & $10^{4} \mathrm{~m}^{2}$ & 1394.306 & 8000 & 65.2 & 1134.004 \\
\multirow{2}{*}{ Output } & approved area & $10^{8} \mathrm{CNY}$ & 462.046 & 3049.83 & 24.22 & 458.970 \\
& GDP & $10^{8} \mathrm{CNY}$ & 191.026 & 2922.612 & 0.05 & 409.761 \\
\hline
\end{tabular}

As the ETDZs were constructed at different times, this study used 115 national ETDZs (including Shanghai, Dalian and Qingdao, etc.) active between 2011 and 2017 as the research objects to balance the number of samples and research time scale. The number of ETDZs located in China's eastern, central, and western regions were 61, 31, and 23, respectively. The data came from the China Development Zones Yearbook, China Development Zones Audit Announcement Catalogue (2018 Edition), and websites related to Development Zones. In addition, this article also supplemented and revised data with the statistical yearbook and statistical bulletin of the ETDZs.

\subsection{Methods}

\subsubsection{Three-Stage DEA Model}

The study used a three-stage DEA model to estimate the operating efficiency of national ETDZs in the period 2011-2017. Three-stage DEA models are generally used in research into the effectiveness of multiple input and output decision-making units. They consider the influence of random noise and environmental factors, and can reflect the real efficiency of decision-making units better than the traditional DEA model [30-32]. The basic idea of the three-stage DEA model is described below.

First stage: traditional DEA model

In the first stage, the original input-output data is used for the initial efficiency evaluation. There are $\mathrm{n}$ decision-making units, and each decision-making unit corresponds 
to $\mathrm{m}$ outputs and $\mathrm{q}$ inputs. The efficiency value of each decision-making unit is calculated as follows [30]:

$$
\begin{gathered}
\rho^{*}=\operatorname{Min} \rho \\
\text { s.t. } \sum_{j=1}^{n} \lambda_{j} x_{i j} \leq \rho x_{i k} ; \sum_{j=1}^{n} \lambda_{j} y_{r j} \geq y_{r k} ; \sum_{j=1}^{n} \lambda_{j}=1 ; \\
\lambda \geq 0 ; j=1,2, \ldots, n ; i=1,2, \ldots, m ; r=1,2, \ldots, q
\end{gathered}
$$

where $\rho^{*}$ is the efficiency value; $\lambda_{j}$ is the linear coefficient of decision-making unit $j ; x_{i j}$ is the input $i$ of decision-making unit $j$; and $y_{r j}$ is the output $r$ of decision-making unit $j$. Based on the traditional DEA model, the slack variables of the decision-making unit can be obtained.

Second stage: SFA model

In the second stage the model is mainly concerned with slack variables that are affected by random errors and environmental factors. By means of SFA regression, the influence of environmental factors and random noise in efficiency measurement is eliminated. The paper constructs the SFA model [32] as follows:

$$
S_{i j}=F^{i}\left(z_{j} ; \beta^{i}\right)+v_{i j}+\mu_{i j} ; j=1,2, \ldots, n ; i=1,2, \ldots, m
$$

where $S_{i j}$ is the slack variable of decision-making unit $j$ input $i ; F^{i}$ is the frontier function corresponding to the slack variable; $z_{j}$ is the environmental variable of decision-making unit $j ; \beta^{i}$ is the parameter to be estimated; $v_{i j}$ is random error; $\mu_{i j}$ is management inefficiency; and $v_{i j}$ and $\mu_{i j}$ are independent of each other. The values of $v_{i j}$ and $\mu_{i j}$ are estimated according to the parameters, and used to effectively adjust the input slack variable. The specific treatment is as follows [32]:

$$
x_{i j}^{*}=x_{i j}+\left[\max \left(z_{j} \beta^{i}\right)-z_{j} \beta^{i}\right]+\left[\max \left(v_{i j}\right)-v_{i j}\right]
$$

where $x_{i j}^{*}$ is the adjusted input; $x_{i j}$ is the original input; $\left[\max \left(z_{j} \beta^{i}\right)-z_{j} \beta^{i}\right]$ describes the adjustment of the external environment of the decision-making unit; and $\left[\max \left(v_{i j}\right)-v_{i j}\right]$ describes the adjustment of the random noise of the decision-making unit. After the above adjustments, each decision-making unit has the same random noise and environmental factor, thereby eliminating the influence of random noise and environmental factors on efficiency measurement.

Third stage: the traditional DEA model

The original input value $x_{i j}$ was replaced with the adjusted input value $x_{i j}^{*}$, and the output value remained unchanged. The DEA model was then used to estimate the efficiency without the influence of environmental and random factors.

\subsubsection{Markov Chain Method}

The Markov chain method was used to reveal the dynamic evolution of the operating efficiency of the ETDZ. A transition probability matrix was used to reflect upward or downward mobility of the operating efficiency of each ETDZ [33,34]. To do this, the operating efficiency of the ETDZ was first discretized into four state types according to quartiles. Then, the probability of various state types was calculated, and the Markov transition probability matrix was built (Table 2). The formula is calculated as follows:

$$
P_{m n}=\frac{K_{m n}}{K_{m}}
$$

where $P$ is the probability, $K$ is the number of ETDZs, $m$ is the state type at time $\mathrm{t}$, and $n$ is the state type at time $t+1$. 
Table 2. Markov transition probability matrix.

\begin{tabular}{ccccc}
\hline $\mathbf{t} \backslash \mathbf{t}+\mathbf{1}$ & $\mathbf{1}$ & $\mathbf{2}$ & $\mathbf{3}$ & $\mathbf{4}$ \\
\hline 1 & $\mathrm{P}_{11}$ & $\mathrm{P}_{12}$ & $\mathrm{P}_{13}$ & $\mathrm{P}_{14}$ \\
2 & $\mathrm{P}_{21}$ & $\mathrm{P}_{22}$ & $\mathrm{P}_{23}$ & $\mathrm{P}_{24}$ \\
3 & $\mathrm{P}_{31}$ & $\mathrm{P}_{32}$ & $\mathrm{P}_{33}$ & $\mathrm{P}_{34}$ \\
4 & $\mathrm{P}_{41}$ & $\mathrm{P}_{42}$ & $\mathrm{P}_{43}$ & $\mathrm{P}_{44}$ \\
\hline
\end{tabular}

\subsubsection{Coupling Coordination Degree Mode}

ETDZs are closely connected with their surrounding areas in the process of development. ETDZs have spillover effects on cities, and cities also exert a supporting effect on the development of ETDZs. Drawing lessons from the concept of physical capacity coupling, a coupling coordination degree model was constructed to estimate the relationship between ETDZ operating efficiency and urban economic development, so as to analyze the degree of interaction and dynamic coupling coordination between the two. The formula is expressed as follows:

$$
\begin{gathered}
C=\sqrt{\left[\left(U_{1} \times U_{2}\right) /\left(\frac{U_{1}+U_{2}}{2}\right)^{2}\right]} \\
T=\alpha U_{1}+\beta U_{2} \\
D=\sqrt{C \times T}
\end{gathered}
$$

where $U_{1}$ is the operating efficiency of the ETDZ; $U_{2}$ is the urban economic growth index, which in this article is characterized by GDP growth rate; $C$ is the coupling degree; $D$ is the coupling coordination degree; $\alpha$ and $\beta$ are index weight, and $\alpha=\beta=0.5$ in this paper; and $T$ is the comprehensive evaluation index.

\section{Results}

\subsection{Evolution of Input and Output Indicators in ETDZs, 2011-2017}

Figure 1 displays the evolution of the average input indicators in China's ETDZs from 2011 to 2017. According to Figure 1a, the average actual utilization of foreign capital in ETDZs across the whole country showed a fluctuating increasing trend between 2011 and 2017. The average foreign capital investment in ETDZs increased from 2.3 billion CNY in 2011 to 2.5 billion CNY in 2017. In 2011, the average actual utilization of foreign capital in the eastern region (3.4 billion CNY) was 2.62 times that in the central region (1.3 billion CNY) and 4.25 times that in the western region ( 800 million CNY). However, by 2017, the average actual utilization of foreign capital in eastern ETDZs was reduced to 2.9 billion $\mathrm{CNY}$, which was 1.07 times that in the central region (2.7 billion CNY) and 2.64 times that in the western region (1.1 billion CNY). As a result of the further deepening of China's opening-up policy, the foreign investment environment in the country's central and western regions has improved, which has gradually reduced regional differences in the actual utilization of foreign capital in ETDZs. As shown in Figure 1b, the average total import volume of China's ETDZs was relatively stable during the study period. During 2011-2016, the average total import volume of ETDZs declined, followed by a significant increase in 2017. Compared with the central and western areas, the average import value in eastern ETDZs was much higher. In 2017, the average total import volume in eastern China's ETDZs (30.1 billion CNY) was 5.9 times that in the central region (5.1 billion $\mathrm{CNY}$ ) and 11.15 times that in the western region (2.7 billion CNY), showing significant regional differences. 


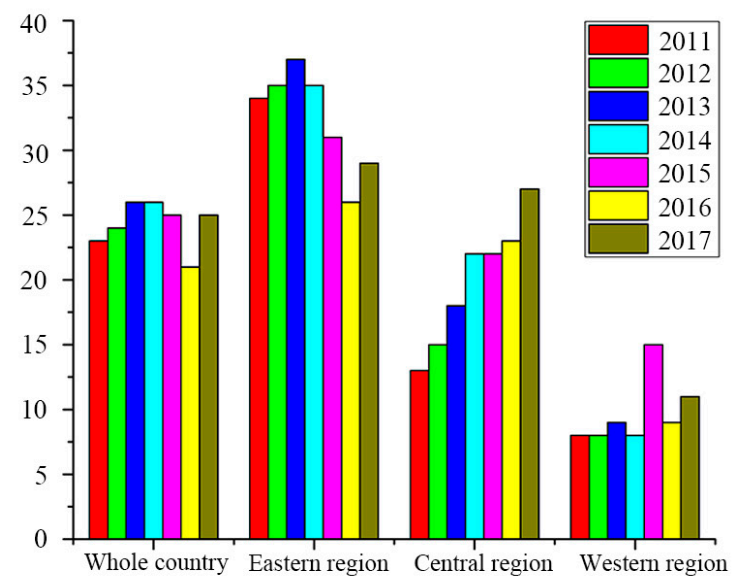

(a) Actual utilization of foreign capital $\left(10^{8} \mathrm{CNY}\right)$

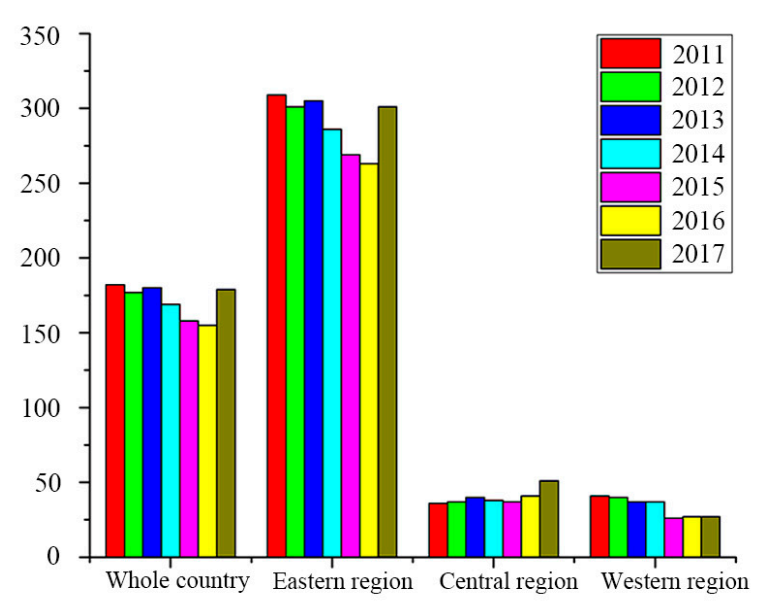

(b) Total import volume $\left(10^{8} \mathrm{CNY}\right)$

Figure 1. The evolution of the average input indicators of ETDZs: (a) actual utilization of foreign capital, (b) total import volume.

Figure 2 shows the evolution of the average output indicators in China's ETDZs from 2011 to 2017. The average GDP per ETDZ showed a continuous upward trend over the course of the research period (Figure 2a). The GDP of China's ETDZs as a whole increased from 33.6 billion CNY to 55.7 billion CNY during the research period. ETDZs in eastern China had the highest GDP, followed by those in central China, and those in western China had the lowest. However, in terms of GDP growth rate, the ETDZs in western China had the highest average annual growth rate $(11.49 \%)$, followed by central China $(10.60 \%)$, with eastern China having the lowest $(7.69 \%)$. Although the scale of the GDP of ETDZs in western China was lower than that of the central and eastern regions, its development speed was the fastest. The average total export volume of the ETDZs increased during the study period (Figure $2 b$ ). However, similar to the average total import value, the average export value of ETDZs in the eastern region was shown to be much higher than in the central and western regions, with significant regional differences. In general, the input and output indicators of ETDZs increased to a certain extent over the study period, but the gap between regions was obvious. The current development level of China's ETDZs is characterized by a significant gap, a finding which verified the necessity of exploring the operating efficiency of ETDZs in different regions. It is worth mentioning that although the west's ETDZs were in total far smaller than the eastern ETDZs, their development speed was shown to be faster than that in the eastern region.

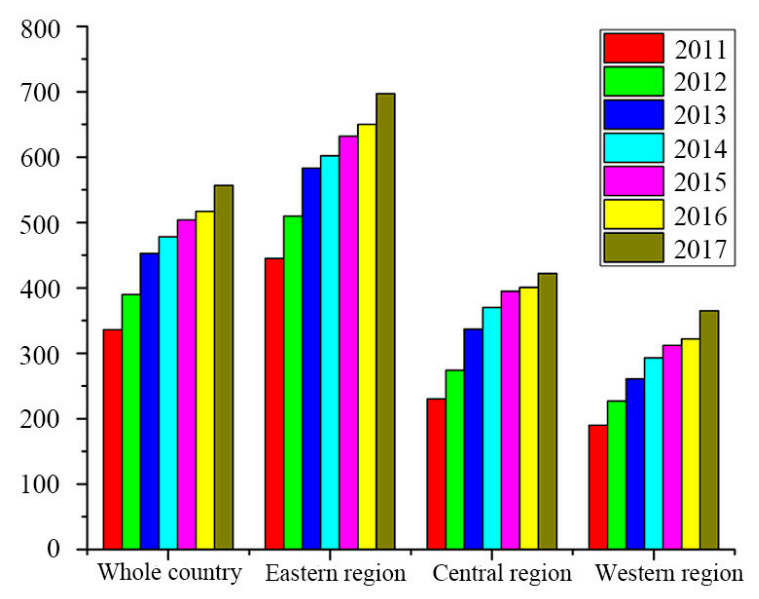

(a) GDP $\left(10^{8} \mathrm{CNY}\right)$

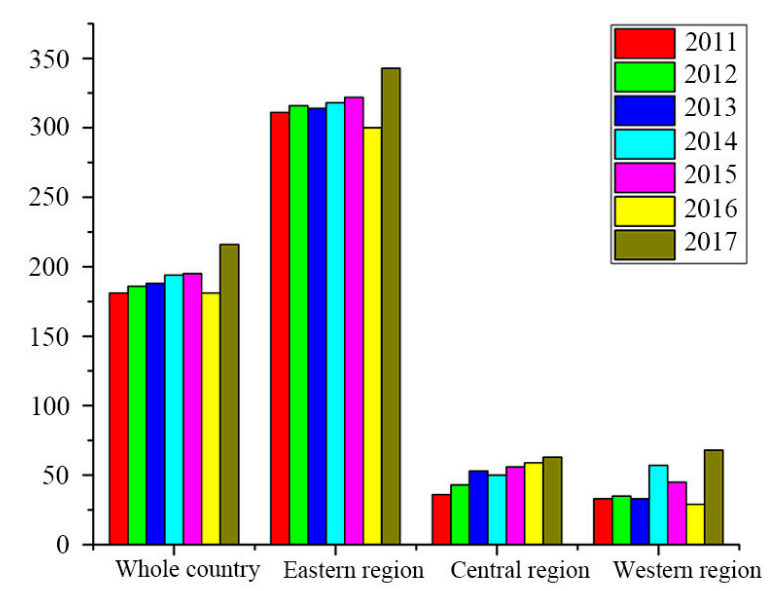

(b) Total export volume $\left(10^{8} \mathrm{CNY}\right)$

Figure 2. The evolution of the average output indicators of ETDZs: (a) GDP, (b) total export volume. 


\subsection{General Characteristics of the Efficiency of China's ETDZs, 2011-2017}

Using a three-stage DEA model and adopting an input-output perspective, this study estimated the operating efficiency of the studied ETDZs. Figure 3 demonstrates the evolution trend of the ETDZs' operating efficiency across the whole country, and the eastern, central and western regions. According to Figure 3, the operating efficiency of China's ETDZs increased in the period 2011-2012, reaching a peak of 0.609 in 2012 and then decreasing from 2012-2017. This suggests that the operating efficiency of China's ETDZs was at a relatively low level in general. In terms of regional distribution, ETDZs in western China had the highest operating efficiency, and ETDZs in central China had the lowest efficiency. The operating efficiency of ETDZs in the western region was found to be higher than the national average. However, compared with western China, the operation efficiency was lower in the eastern and central regions, leaving room for improvement.

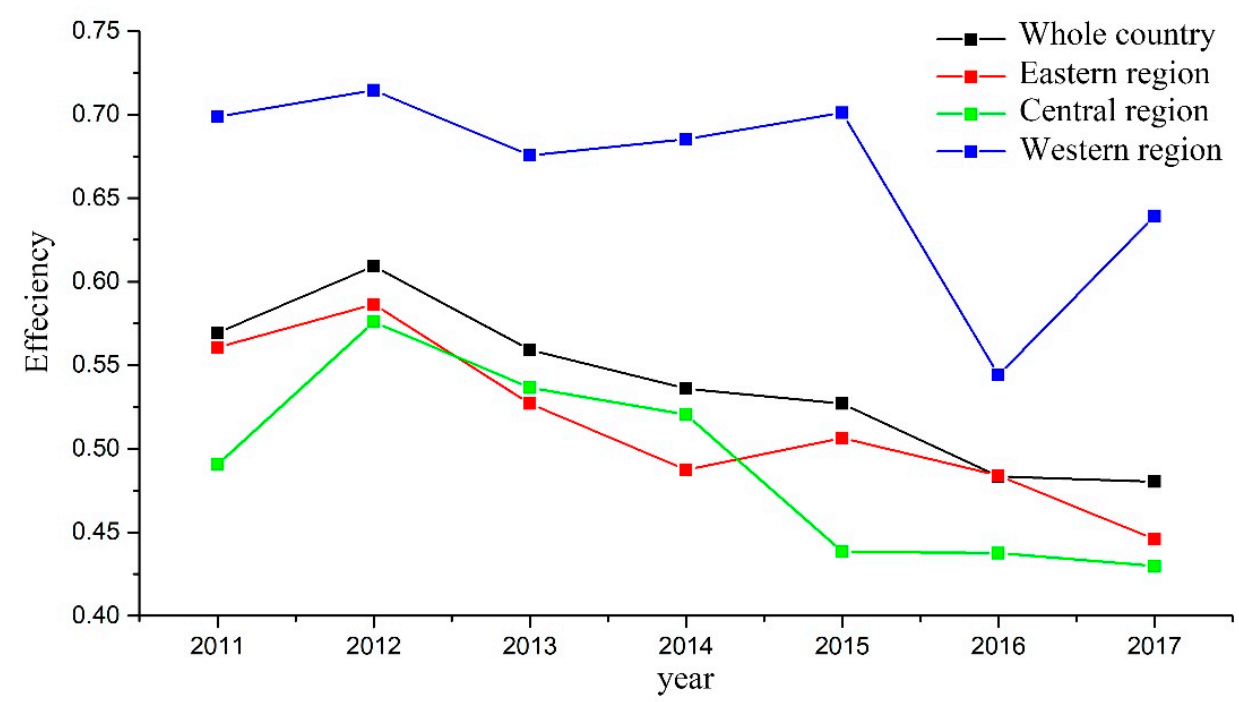

Figure 3. The Evolution of the ETDZ's Operating Efficiency.

The regional differences in operating efficiency suggest that the development of national ETDZs is still at a factor-driven phase and depends on the scale of factor input rather than technical improvement. The main reason for this may be that local governments privilege speed over quality with respect to economic development during the construction of ETDZs. In order to obtain higher economic outputs, they may have blindly expanded their ETDZs, failing to pay adequate attention to the quality and technical level of foreign investment and to carry out scientifically grounded development plans. This extensive development model may have increased the economic outputs of the ETDZs to a certain extent, but it also has resulted in unnecessary land use and inefficient funds allocation in the ETDZs. Therefore, although the input and output scale of ETDZs in eastern and central China is higher than that in western China (Figures 1 and 2), the operating efficiency is far lower.

The box diagram presented in Figure 4 displays the overall characteristics of the operating efficiency of China's ETDZs. It shows that the number of ETDZs with optimal efficiency declined over the study period. In 2011, there were 19 ETDZs with optimal operating efficiency. By 2017, the number of ETDZs with optimal operating efficiency had decreased to 13. In addition, the number of ETDZs with an operating efficiency of less than 0.5 increased from 56 in 2011 to 72 in 2017. Despite the increase in outputs (Figure 2), the operating efficiency of many ETDZs decreased during the research period. The results presented in Figure 4 also indicate that the concentrated distribution interval of ETDZs' operating efficiencies evolved from $0.2-0.7$ to $0.1-0.8$, gradually spreading towards both ends. Thus, this paper concludes that during the research period, the operating efficiency of China's ETDZs decreased, and the gap between different ETDZs became more pronounced. 


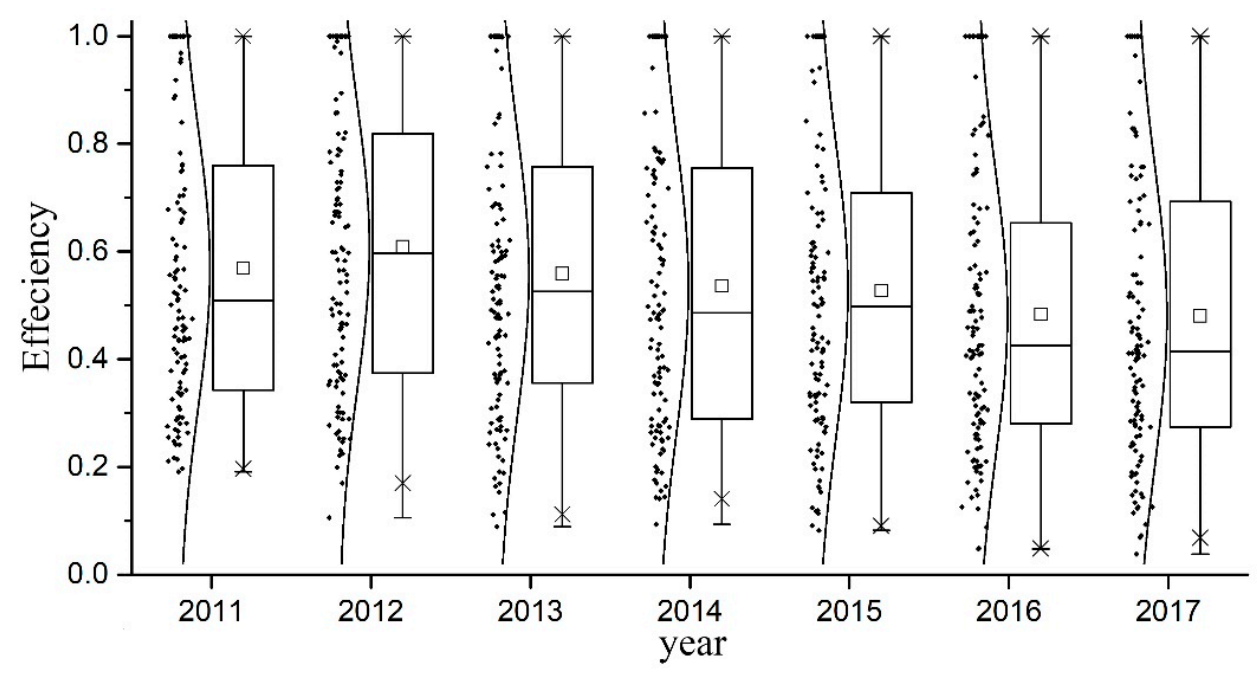

Figure 4. The box diagram of the ETDZ's operating efficiency.

\subsection{The Evolution Characteristics of the Efficiency of National ETDZs, 2011-2017}

This study divided China's ETDZs into four types according to their operating efficiency, with type 4 representing high efficiency and types 3,2, and 1 representing medium high, medium-low and low efficiency respectively. Situations where an EDTZ moved from a lower operating efficiency value to a higher one is defined as an upward transfer, and the opposite is defined as a downward transfer. Table 3 shows the probability of the occurrence of these two operating efficiency transfer types in relation to China's ETDZs. These results reveal that the probability that the operating efficiency of the ETDZ remained stable was the highest, at 0.670 . Moreover, the probability of operating efficiency exhibiting a downward transfer was found to be higher than the probability of it registering an upward transfer $(0.194>0.136)$. This indicates that despite the prevalent tendency for operating efficiency to remain stable across the study period, a downward transfer trend could be discerned (i.e., when transfers occurred, they tended to be downward). With respect to regional distribution, ETDZs in the central region maintained the most stable operating efficiency levels, retaining the same efficiency type (0.704); ETDZs in western China displayed the highest fluctuation in operating efficiency, with the probability of downward transfer and upward transfer both being higher than those in the eastern and central regions $(0.1522>0.1339>0.1290 ; 0.2246>0.1967>0.1667)$.

Table 3. Probability of different types of operating efficiency transfers in China's ETDZs.

\begin{tabular}{cccc}
\hline & Upward & Stable & Downward \\
\hline Whole country & 0.1362 & 0.6696 & 0.1942 \\
Eastern region & 0.1339 & 0.6694 & 0.1967 \\
Central region & 0.1290 & 0.7043 & 0.1667 \\
Western region & 0.1522 & 0.6232 & 0.2246 \\
\hline
\end{tabular}

Table 4 displays the Markov transition probability matrix for operating efficiency of ETDZs in the whole country and various regions. According to the Markov transition probability matrix the following dynamic evolution characteristics of the operating efficiency of ETDZs can be obtained.

First, the diagonal probability in the probability matrix was generally higher than the off-diagonal probability. Except for P11 in the west region, the diagonal probabilities of the matrix were all higher than 0.5 , which further indicates strong stability in the operating efficiency of China's ETDZs. The outlier in the western region may be caused by too few ETDZs of this type. 
Table 4. Markov transition probability matrix of the operating efficiency of China's ETDZs.

\begin{tabular}{ccccccc}
\hline \multirow{5}{*}{ Whole country } & $\mathbf{t} \backslash \mathbf{t}+\mathbf{1}$ & $\mathbf{n}$ & $\mathbf{1}$ & $\mathbf{2}$ & $\mathbf{3}$ & $\mathbf{4}$ \\
\hline & 1 & 87 & 0.7242 & 0.2413 & 0.0115 & 0.0230 \\
& 2 & 258 & 0.1395 & 0.6899 & 0.1125 & 0.0581 \\
Eastern region & 3 & 174 & 0.0230 & 0.2529 & 0.5747 & 0.1494 \\
& 4 & 171 & 0.0058 & 0.0819 & 0.2047 & 0.7076 \\
& 1 & 50 & 0.7200 & 0.2600 & 0.0200 & 0 \\
Central region & 2 & 156 & 0.1410 & 0.7115 & 0.0962 & 0.0513 \\
& 3 & 76 & 0.0262 & 0.2763 & 0.5396 & 0.1579 \\
& 1 & 84 & 0.0119 & 0.0833 & 0.2262 & 0.6786 \\
Western region & 2 & 28 & 0.8571 & 0.1429 & 0 & 0 \\
& 4 & 70 & 0.1143 & 0.6857 & 0.1714 & 0.0286 \\
& 1 & 26 & 0 & 0.2742 & 0.6290 & 0.0968 \\
& 3 & 9 & 0.3334 & 0.4444 & 0 & 0.7693 \\
& 3 & 32 & 0.1875 & 0.5938 & 0.0625 & 0.1562 \\
& 4 & 61 & 0.0556 & 0.1667 & 0.5556 & 0.2221 \\
& 4 & 0 & 0.0820 & 0.0327 & 0.7213 \\
\hline
\end{tabular}

Second, the high efficiency downward transfer probability of ETDZs in the western region $(\mathrm{P} 43=0.037)$ was much lower than that in the whole country, the eastern and the central areas $(\mathrm{P} 43=0.2047,0.2262,0.1538)$ respectively. In addition, the low efficiency downward transfer probability of ETDZs in the western area was higher than other areas, while their upward transfer probability was lower. This indicates that ETDZs with high operating efficiency in the western region enjoyed a better development trend than in other regions, while ETDZs with low operating efficiency demonstrated the opposite.

Third, the operating efficiency transfer type of ETDZs displayed a "convergence phenomenon," whereby the upward transfer probability of ETDZs with low operating efficiency was higher than those with high operating efficiency (P12 > P34), and the downward transfer probability of ETDZs with high operating efficiency was also higher than those with low operating efficiency (P43 > P21).

Fourth, the operating efficiency of ETDZs in adjacent years rarely achieved leapfrog transfer. The probability of achieving a leap-forward transfer of operational efficiency was below 0.1 with the only exception being in the western region. This confirms that between two consecutive years, the probability of a leapfrog transfer of operating efficiency was extremely small.

\subsection{The Coupling Coordination Relationship between the ETDZs' Operating Efficiency and Urban Economic Development}

Nationally, ETDZs have acted as a window for China to open up to the outside world [2]. The government established ETDZs to attract foreign investment and advanced technology, and in this way promote regional economic development. This research adopted a coupling coordination degree model to explore the coupling coordination relationship between operating efficiency of national ETDZs and urban economic development.

Based on cluster analysis, the coupling coordination relationship between operating efficiency of ETDZs and urban economic growth was divided into four stages, comprising low $(0<\mathrm{D} \leq 0.3)$, medium-low $(0.3<\mathrm{D} \leq 0.5)$, medium-high $(0.5<\mathrm{D} \leq 0.8)$, and high $(0.8<\mathrm{D} \leq 1)$ coupling coordination stages. According to Table 5 , the degree of coupling coordination between the operating efficiency of national ETDZs and urban economic development was characterized by a trend of increasing fluctuation. The degree increased from 0.6388 in 2011 to 0.7157 in 2016, and then decreased to 0.6609 in 2017, indicating that the coupling coordination relationship between operating efficiency of ETDZs and urban economic development was not stable. On the whole, from 2011 to 2017, the relationship occupied a medium-high coupling coordination stage. As spatial units within cities, ETDZs 
could be seen to maintain a close relationship with the development of cities through operating efficiency.

Table 5. The coupling coordination relationship between the efficiency of ETDZs and urban economic development.

\begin{tabular}{ccccc}
\hline & $\mathbf{U}_{\mathbf{1}}$ & $\mathbf{U}_{\mathbf{2}}$ & $\mathbf{C}$ & $\mathbf{D}$ \\
\hline 2011 & 0.4677 & 0.4852 & 0.8940 & 0.6388 \\
2012 & 0.5629 & 0.4107 & 0.9277 & 0.6573 \\
2013 & 0.5162 & 0.4065 & 0.9172 & 0.6362 \\
2014 & 0.4884 & 0.4167 & 0.9089 & 0.6272 \\
2015 & 0.4848 & 0.5684 & 0.9073 & 0.6815 \\
2016 & 0.4575 & 0.7370 & 0.8724 & 0.7157 \\
2017 & 0.4598 & 0.5336 & 0.9056 & 0.6609 \\
\hline
\end{tabular}

Figure 5 presents the evolution of the coupling coordination relationship between ETDZs and urban economic growth in various regions. According to Figure 5, the change in coupling coordination in China's eastern and central regions showed a trend of increasing fluctuation, from 0.6175 and 0.6028 in 2011 to 0.6707 and 0.6523 in 2017, respectively. This trend is consistent with the characteristic of the operating efficiency of ETDZs, presenting an M-type change trend in each region. From the perspective of regional differences, the coupling coordination relationship in western China remained higher than in eastern and central China over the course of the research period, although the gap between regions gradually decreased.

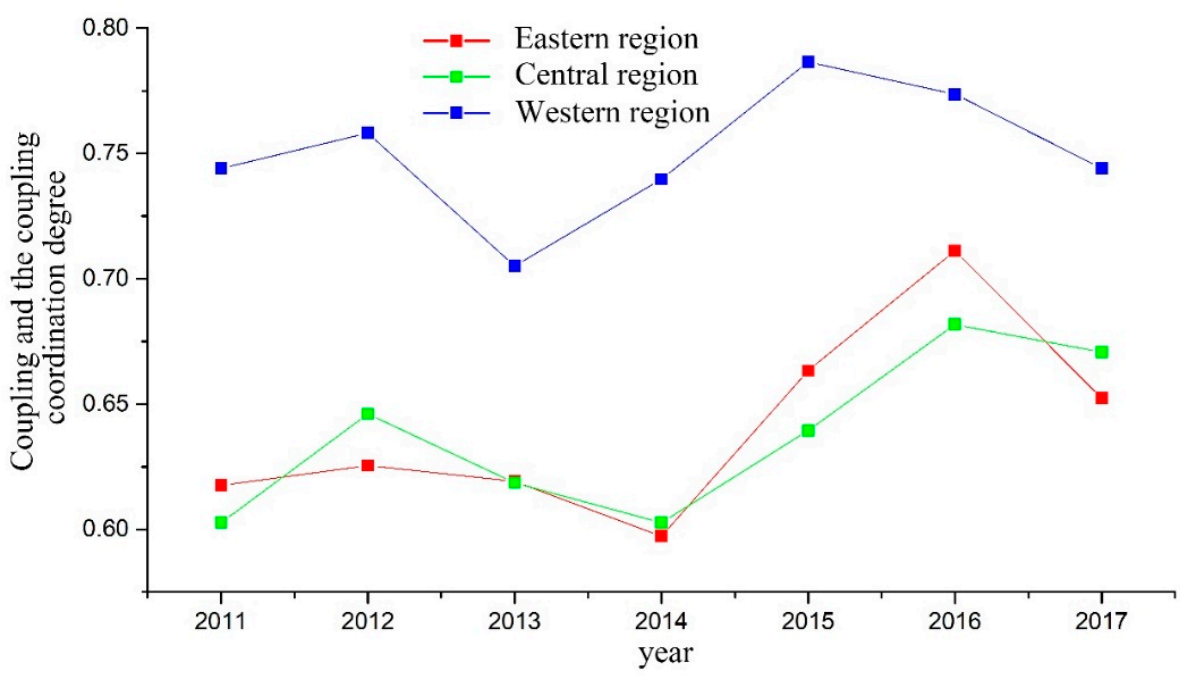

Figure 5. The evolution of the coupling coordination relationship between the operating efficiency of ETDZs and urban economic development in various regions.

Across the whole country, as well as across separate regions, the coupling coordination stage showed some improvement over the study period, although it did not enter the medium-high coupling coordination stage. The stages of the whole country and the eastern and central areas were much lower than that of western China. The operating efficiency of ETDZs in the whole country and the east and the middle areas generally lagged behind the level of urban economic growth. In these regions, we found that urban economic growth was mainly promoted by changes in the scale of the ETDZs, a factor which was not strongly affected by the operating efficiency of the ETDZ—-for this reason, these regions exhibit a low degree of coupling coordination. For the western region, the area of ETDZs tended to be smaller but more efficient. The findings suggest that the efficiency of ETDZs can significantly improve the level of urban economic development, and at the same time, 
urban economic development can also improve the operating efficiency of the ETDZ, achieving a coordinated improvement between the two.

\section{Conclusions and Discussion}

This article used a three-stage DEA model to estimate the operating efficiency of China's ETDZs and identify their dynamic evolutionary trends. It then introduced a coupling coordination degree model to investigate the mutual influence of operation efficiency of ETDZs and urban economic development. In addition, the country was divided into eastern, central, and western regions so as to discern inter-regional diversity of development of national ETDZs. The following conclusions can be drawn from the results.

The operating efficiency of China's ETDZs was found to generally exist at a low level, and to present a decreasing trend during the course of the research period. The unevenness among China's ETDZs in terms of their operating efficiency appeared particularly significant. The operating efficiency of ETDZs was highest in western China, second highest in central China, and lowest in eastern China. The operating efficiency of ETDZs in the eastern and central areas left much room for improvement. The Markov chain transition probability matrix showed that operating efficiency of China's ETDZs was stable, although a trend towards downward transfers was identified. ETDZs in the eastern region had the most stable operating efficiency, while those in the western region displayed the greatest volatility. There existed "convergence phenomena" in the type of transition experienced by ETDZs in their operation efficiency, and the results reinforce the fact that realizing leapfrog development was extremely difficult.

This article found that the degree of coupling coordination between the operation efficiency of ETDZs and urban economic development was characterized by increasing volatility during the research period, and generally occupied a medium-high coupling coordination stage. The coupling coordination degree presented an M-type trend in all of China's regions, although this value was higher in the western than in the eastern and central regions. Moreover, the findings illustrate that when an ETDZ's operating efficiency was high, coordination with urban economic growth significantly improved. This can be attributed to the fact that high-efficiency ETDZs bring about strong spatial spillover effects, exerting a more significant influence on regional economic growth as a result $[8,15]$.

As the flagship of China's reform and opening up policy, ETDZs play an important role in regional economic growth in attracting investment, promoting exports, and technological progress [10]. Existing studies have concluded that land use efficiency and output efficiency of enterprises in ETDZs with better management are significantly higher than those in other areas, and this spillover effect may also promote urban construction and economic development around the ETDZ [13-15]. This paper verifies the above research conclusions to a certain extent, but only ETDZs with high operating efficiency appeared to have a significant role in promoting these effects. Unfortunately, from the perspective of operating efficiency, development of China's ETDZs was revealed here as not being ideal. Especially in the eastern and central parts of China, there was great potential for improvement in terms of operating efficiency of ETDZs. At present, most ETDZs in China remain in a factor-driven development stage, and expansion in their scale often comes at the expense of efficiency. Similar to the conclusions of existing studies, this article also found that due to the low management capacity of some ETDZs, both land and funds cannot be used effectively, which is not conducive to the sustainability of the development of ETDZs and adjacent cities $[20,21]$. Based on the above statement, it can be suggested that the government should pay greater attention to the overall efficiency and growth quality in the sustainable development of the country's ETDZs. All of this points to the fact that ETDZs should not blindly expand in scale, especially with respect to the scale of foreign capital. Instead, such zones should be supported by a scientific industrial development plan. Based on the industrial foundation and competitive advantages, ETDZs should reduce the scope of foreign capital in their industrial activities, and rather focus on the quality of foreign investment. In addition, the government needs to increase inputs in technological 
innovation platforms and strengthen the allocation and utilization of resources to improve the contribution of technological progress to the operating efficiency of ETDZs.

This research has the following limitations. Incomplete statistical data on ETDZs and the lag in the publication time have had a certain impact on the index selection and time span of this research, and the factors affecting the ETDZ's efficiency have not been analyzed in depth. In addition, the interactive relationship between ETDZs and cities is intricate. Elucidating the influence mechanism between ETDZs and cities is worthy of in-depth analysis in future research.

Author Contributions: Conceptualization, S.G. and R.W.; methodology, J.Z.; software, J.Z.; validation, S.G. and R.W.; formal analysis, J.Z.; investigation, X.M.; resources, X.M.; data curation, X.M.; writing—original draft preparation, S.G.; writing—review and editing, S.G.; visualization, J.Z.; supervision, R.W.; project administration, R.W.; funding acquisition, R.W. All authors have read and agreed to the published version of the manuscript.

Funding: This research was funded by the National Natural Science Foundation of China (42001147), the Construction of National Science and Technology Think Tank of Guangzhou (SXK20210401054).

Institutional Review Board Statement: Not applicable.

Informed Consent Statement: Not applicable.

Data Availability Statement: Data is contained within the article.

Conflicts of Interest: The author declares no conflict of interest.

\section{References}

1. Ge, W. Special Economic Zones and the Economic Transition in China; World Scientific: Singapore, 1999.

2. Yeung, Y.M.; Lee, J.; Kee, G. China's special economic zones at 30. Eurasian Geogr. Econ. 2009, 50, 222-240. [CrossRef]

3. Zeng, D.Z. Global experiences with special economic zones: Focus on China and Africa. Soc. Sci. Res. Netw. 2015, 7240, 1-23.

4. Chu, D. The special economic zones of China and their impact on its economic development. Jung-Dong Park. China Q. 1999, 1, 496-497.

5. Wei, Y.H.D.; Leung, C.K. Development zones, foreign investment, and global city formation in Shanghai. Growth Chang. 2010, 36, 16-40. [CrossRef]

6. National Development and Reform Commission; The State Development and Reform Commission. China Development Zone Audit Bulletin Directory. 2018. Available online: https://www.ndrc.gov.cn/xxgk/zcfb/gg/201803/t20180302_961203.html? code $=\&$ state $=123$ (accessed on 26 February 2018).

7. Walcott, S.M. Chinese industrial and science parks: Bridging the gap. Prof. Geogr. 2002, 54, 349-364. [CrossRef]

8. Simon, A.; Lin, S.; Fabrizio, Z. Economic reforms and industrial policy in a panel of Chinese cities. J. Econ. Growth 2013, 21, 1-45.

9. Huang, Z.; He, C.; Zhu, S. Do China's economic development zones improve land use efficiency? The effects of selection, factor accumulation and agglomeration. Landsc. Urban Plan. 2017, 162, 145-156. [CrossRef]

10. Wang, J. The economic impact of special economic zones: Evidence from Chinese municipalities. J. Dev. Econ. 2013, 101, 133-147. [CrossRef]

11. Herlevi, A.A. Economic growth or sowing the seeds of destruction? The role of economic development zones in China. J. Chin. Political Sci. 2017, 22, 675-689. [CrossRef]

12. Huang, Z.; He, C.; Wei, Y.H.D. A comparative study of land efficiency of electronics firms located within and outside development zones in Shanghai. Habitat Int. 2016, 56, 63-73. [CrossRef]

13. Sun, Y.; Ma, A.; Su, H.; Su, S.; Chen, F.; Wang, W.; Weng, M. Does the establishment of development zones really improve industrial land use efficiency? Implications for China's high-quality development policy. Land Use Policy 2020, 90, 104265. [CrossRef]

14. Schminke, A.; Biesebroeck, J.V. Using export market performance to evaluate regional preferential policies in China. Rev. World Econ. 2013, 149, 343-367. [CrossRef]

15. Luo, D.; Liu, Y.; Wu, Y.; Zhu, X.; Jin, X. Does development zone have spillover effect in China? J. Asia Pac. Econ. 2015, 20, 489-516. [CrossRef]

16. Yeh, A.G.-O.; Wu, F. The new land development process and urban development in Chinese cities. Int. J. Urban Reg. Res. 2010, 20, 330-353. [CrossRef]

17. Cartier, C. 'Zone Fever', the arable land debate, and real estate speculation: China's evolving land use regime and its geographical contradictions. J. Contemp. China 2001, 10, 445-469. [CrossRef]

18. Zhang, J. Interjurisdictional competition for FDI: The case of China's "development zone fever". Reg. Sci. Urban Econ. 2011, 41, 145-159. [CrossRef] 
19. Wei, Y.H.D. Zone fever, project fever: Development policy, economic transition, and urban expansion in China. Geogr. Rev. 2015, 105, 156-177. [CrossRef]

20. $\mathrm{Hu}, \mathrm{X}$. A study of sustainable development of China's state-level economic and technological development zones. China World Econ. 2005, 13, 92-106.

21. Zhang, H.; Sonobe, T. Development of science and technology parks in China, 1988-2008. Economics 2011, 5, 1-25. [CrossRef]

22. Liu, Y.; Li, J.; Yang, Y. Strategic adjustment of land use policy under the economic transformation. Land Use Policy 2018, 74, 5-14. [CrossRef]

23. Wang, Z.; Feng, C. A performance evaluation of the energy, environmental, and economic efficiency and productivity in China: An application of global data envelopment analysis. Appl. Energy 2015, 147, 617-626. [CrossRef]

24. Zhu, J. From land use right to land development right: Institutional change in China's urban development. Urban Stud. 2004, 41, 191-220. [CrossRef]

25. Chen, C.J.; Wu, H.L.; Lin, B.W. Evaluating the development of high-tech industries: Taiwan's science park. Technol. Forecast. Soc. Chang. 2006, 73, 452-465. [CrossRef]

26. Lin, G.C.S.; Ho, S.P.S. The State, Land System, and Land Development Processes in Contemporary China. Ann. Assoc. Am. Geogr. 2005, 95, 411-436. [CrossRef]

27. Sun, C.C. Evaluating and benchmarking productive performances of six industries in Taiwan Hsin Chu Industrial Science Park. Expert Syst. Appl. 2011, 38, 2195-2205. [CrossRef]

28. Xu, Y.; Tang, B.S.; Chan, E.H.W. State-led land requisition and transformation of rural villages in transitional China. Habitat Int. 2011, 35, 57-65. [CrossRef]

29. Benwu, X. The research on the technological innovation efficiency of Chinese industries. Sci. Res. Manag. 2011, 32, 10.

30. Charnes, A.; Cooper, W.W.; Rhodes, E. Measuring the efficiency of decision making units. Eur. J. Oper. Res. 1978, 2, 429-444. [CrossRef]

31. Banker, R.D.; Charnes, A.; Cooper, W.W. Some models for estimating technical and scale inefficiencies in data envelopment analysis. Manag. Sci. 1984, 30, 1078-1092. [CrossRef]

32. Freid, H.O.; Lovell, C.A.K.; Schmidt, S.S.; Yaisawaring, S. Accounting for environmental effects and statistical noise in data envelopment analysis. J. Product. Anal. 2002, 17, 157-174. [CrossRef]

33. Gallo, J.L. Space-time analysis of GDP disparities among European regions: A Markov chains approach. Int. Reg. Sci. Rev. 2004, 27, 138-163. [CrossRef]

34. Liao, F.H.F.; Wei, Y.H.D. Dynamics, space, and regional inequality in provincial China: A case study of Guangdong province. Appl. Geogr. 2012, 35, 71-83. [CrossRef] 